\title{
Estrategias de innovación para el desarrollo y la resiliencia de ciudades medias*
}

\author{
Ricardo Méndez Gutiérrez del Valle \\ Centro de Ciencias Humanas y Sociales. CSIC \\ Instituto de Economía, Geografía y Demografía \\ ricardo.mendez@cchs.csic.es
}

Recepción: mayo de 2012
Aceptación: enero de 2013

\section{Resumen}

Las ciudades de nuestro entorno se enfrentan a una competencia creciente derivada de la globalización, así como al impacto de una crisis sistémica que cuestiona determinados modelos de crecimiento anteriores. Al objetivo de incrementar el esfuerzo de innovación económica y social se suma ahora el de utilizar tales estrategias para recuperar ciudades enfrentadas a procesos de declive. Aunque la mayoría de estudios sobre innovación, conocimiento o creatividad se centran en las grandes metrópolis, las ciudades medias despiertan un creciente interés, tanto desde la perspectiva de la cohesión territorial como por ofrecer ejemplos recientes de ciudades que superaron procesos de declive a partir de estrategias innovadoras. El artículo aporta una reflexión teórica sobre los conceptos de ciudad innovadora y resiliencia urbana, identifica sus principales características, ofrece una interpretación de las claves que explican la desigual trayectoria de las ciudades medias y propone algunas políticas para favorecer la innovación.

Palabras clave: innovación económica y social; crisis; resiliencia urbana; ciudades medias.

\section{Resum. Estratègies d'innovació per al desenvolupament i la resiliència de ciutats mitjanes}

Les ciutats del nostre entorn s'enfronten a una competència creixent derivada de la globalització, com també a l'impacte d'una crisi sistèmica que qüestiona determinats models de creixement anteriors. A l'objectiu d'incrementar l'esforç d'innovació econòmica i social, s'hi afegeix el d'utilitzar aquestes estratègies per recuperar ciutats enfrontades a processos de declivi. Encara que la majoria d'estudis sobre innovació, coneixement o creativitat se centren en grans metròpolis, les ciutats mitjanes desperten un interès creixent, tant des de la perspectiva de la cohesió territorial com perquè ofereixen exemples de ciutats que van superar processos de declivi a partir d'estratègies innovadores. L'article aporta una reflexió teòrica sobre els conceptes de ciutat innovadora i resiliència urbana, n'identifica les característiques principals, fa una interpretació de les claus que expliquen la trajectòria desigual de les ciutats mitjanes i proposa polítiques per afavorir la innovació.

Paraules clau: innovació econòmica i social; crisi; resiliència urbana; ciutats mitjanes.

* El artículo se integra en el proyecto de investigación financiado por el Plan Nacional de I + D + i sobre Efectos socioterritoriales de la crisis económica en las áreas urbanas de España: politicas públicas y estrategias de resiliencia (CS[2012-36170). 
Résumé. Stratégies d’innovation pour le développement et la résilience des villes moyennes

Les villes de notre région sont confrontées à une concurrence croissante liée à la mondialisation, même qu'à l'impact d'une crise systémique qui remet en question certains modèles de croissance précédents. À l'objectif de renforcer l'effort d'innovation économique et social s'ajoute l'usage de ces stratégies pour récupérer des villes en déclin. Bien que la plupart d'études sur l'innovation, le savoir ou la créativité ont été menées dans des grandes villes et régions métropolitaines, les villes moyennes suscitent un intérêt croissant, tant du point de vue de la cohésion territorial que par fournir des exemples de villes qui ont dépassé le déclin à partir de stratégies innovantes. L'article propose une réflexion théorique sus les concepts de la ville innovante et la résilience urbaine, identifie ses caractéristiques, joue les clés de l'inégale évolution des villes moyennes et propose des politiques pour la promotion de l'innovation.

Mots clé: innovation économique et sociale; crise; résilience urbaine; villes moyennes.

\section{Abstract. Innovation strategies for development and resilience of medium-size cities}

Cities of our area are faced with a raising competition from globalization, as well as with the impact of a systemic crisis that questions some previous growth models. Along with the aim of increasing the effort of economic and social innovation, is important now to use these strategies to overcome the shrinkage of some cities. Although most studies on innovation, knowledge or creativity have been focused on large cities and metropolitan areas, the medium-sized cities shows a growing interest, both from the perspective on territorial cohesion as to provide recent examples of cities that have surpassed decline processes through innovative strategies. This paper provides a theoretical reflection on the concepts of innovative city and urban resilience, identifies its main features, presents the keys factors to explain the uneven paths of medium-sized cities and propose policies to promote urban innovation.

Keywords: economic and social innovation; crisis; urban resilience; medium-size cities.

\section{Sumario}

Introducción Ciudades medias frente a la crisis:

El discurso sobre la ciudad innovadora: una propuesta desde la perspectiva del desarrollo urbano

Ciudades medias e innovación: de las debilidades genéricas a las potencialidades específicas innovación para la resiliencia urbana

Claves para la construcción de ciudades medias innovadoras y resilientes

Conclusiones

Referencias bibliográficas 


\section{Introducción}

Desde hace más de dos décadas, nuestras ciudades se enfrentan al doble reto que suponen un proceso de globalización dominado por principios neoliberales y otro paralelo de integración en un marco institucional común, donde convergencia y competencia se refuerzan mutuamente y generan nuevos contrastes a diferentes escalas. A ello se une ahora el impacto de una crisis sistémica que, si bien tiene su epicentro en el sistema financiero, desborda sobre el conjunto de la actividad económica y provoca graves costes sociales. También en esto las ciudades muestran un diverso grado de vulnerabilidad y capacidad de respuesta, pues, mientras algunas resisten mejor sus embates, otras se enfrentan a un profundo y prolongado declive que cuestiona su anterior modelo de crecimiento y plantea serias incertidumbres sobre su futuro.

En un contexto fluido como el actual, adquiere especial importancia la capacidad de las ciudades para articular y poner en práctica estrategias que aseguren su competitividad y la generación de empleo, eleven o al menos mantengan la calidad de vida de sus habitantes, promuevan un urbanismo más sostenible, o favorezcan una democracia local más participativa. Se trata de sustituir prácticas ineficientes o ajenas a los intereses de la mayoría de sus ciudadanos aportando soluciones inteligentes, inclusivas y comprometidas, con un objetivo de desarrollo no limitado al plano económico, sino capaz de incorporar las múltiples dimensiones de este concepto.

Son numerosos los documentos de la Comisión Europea que, desde la Agenda de Lisboa (2000) hasta la Estrategia Europa 2020, han insistido en la importancia de incrementar la generación y aplicación de conocimiento, así como la difusión de innovaciones, para avanzar en el objetivo de un crecimiento «inteligente, sostenible e integrador» (Comisión Europea, 2010). El discurso de la innovación ha pasado así a formar parte de la retórica oficial, aunque con significados muy diversos según los casos, que banalizan a menudo su sentido original y obligan a precisar el contenido que se le asigna en propuestas orientadas al desarrollo urbano como la presente.

Los estudios sobre innovación, conocimiento o creatividad se han incorporado a la agenda de los estudios urbanos, pero prestando siempre especial atención a las grandes ciudades-región que cuentan con la mayor cantidad de recursos materiales y humanos para su impulso. No obstante, desde la aprobación de la Estrategia Territorial Europea (1999) y la Agenda Territorial de la Unión Europea (2007), ha aumentado también la atención otorgada a las ciudades medias, tanto por su significado para construir sistemas urbanos más policéntricos y equilibrados, capaces de promover mayor cohesión territorial, como por su potencial función de intermediación con respecto a las áreas rurales. Pero se abre paso ahora otra dimensión menos explorada al constatar la capacidad de algunas de ellas para buscar respuestas innovadoras en planos tan diversos como el empresarial y tecnológico, el de la movilización social o la gestión pública, así como para enfrentarse a la crisis de manera proactiva, aspectos de especial relevancia en el momento presente. 
Con esta perspectiva, el artículo propone una interpretación de carácter teórico a partir de dos líneas de investigación que pueden orientar estrategias adecuadas para el desarrollo de ciudades medias y para la recuperación de aquellas que se enfrentan a procesos de declive. Están, por una parte, los estudios sobre ciudades innovadoras que tienen como referente inicial la bibliografía surgida hace dos décadas en geografía económica sobre los milieux innovateurs, pero reorientada por aquella otra que sobre innovación social ha proliferado en el último decenio. A estos se unen los vinculados a un concepto emergente como el de resiliencia urbana, aplicable a aquellas ciudades que se han enfrentado a un profundo declive derivado de algún shock externo acentuado por bloqueos endógenos y que han logrado revitalizar su economía y regenerar su tejido urbano y social. Los enfoques evolucionistas, atentos a la influencia ejercida por cada trayectoria urbana, junto a los relacionales que destacan la importancia de las redes de actores, así como el peso de las normas, valores, organizaciones y políticas locales que señalan las perspectivas neoinstitucionales, suponen un substrato común que aporta coherencia a planteamientos inicialmente heterogéneos.

El texto se inicia con una referencia al concepto de ciudad innovadora para precisar su significado en la perspectiva de los estudios sobre desarrollo territorial. Destaca luego las debilidades genéricas que a menudo se atribuyen a las ciudades medias frente a las grandes para convertirse en focos de innovación, acentuadas en aquellas sometidas a procesos de declive, junto a algunas oportunidades para superar esas desventajas comparativas. Pero la atención se centra, sobre todo, en identificar posibles claves explicativas de la desigual capacidad de innovación y resiliencia que muestran ciudades de tamaño similar, a partir de ejemplos existentes en la bibliografía internacional y de estudios sobre ciudades españolas publicados en los últimos años, que, aunque escasos en número, ofrecen ya una cierta base para profundizar en esta temática.

\section{El discurso sobre la ciudad innovadora: una propuesta desde la perspectiva del desarrollo urbano}

En un contexto dominado por la aceleración de los procesos y la creciente interdependencia de los territorios, el aumento de los riesgos globales y de las incertidumbres derivadas para un elevado número de ciudades y regiones parece haberse convertido ya en lugar común de esta nueva normalidad que define nuestro tiempo. Tal situación aumenta el valor de la anticipación y la prospectiva, pero sobre todo de la capacidad de adaptación a unos cambios rápidos y profundos, lo que equivale a reconocer la creciente importancia de la innovación y la creatividad como base para encontrar nuevos caminos para el desarrollo.

Aunque el aumento del saber no permita controlar lo imprevisible, sí puede favorecer esa adaptación positiva, lo que explica la reciente multiplicación de metáforas para identificar a aquellas ciudades que parecen mejor dotadas para lograrlo. Conceptos como los de learning cities (OCDE, 2001), creative cities (Hall, 2000; Florida, 2005), intelligent cities (Komninos, 2002), knowledge 
cities (Van Winden et al., 2007) o smart cities (Caragliu et al., 2009) son algunos de los más utilizados en estos últimos años. Más allá de una simple moda o una imagen de marca para sus autores, todos ellos responden a un discurso sobre las claves actuales de la competitividad y el desarrollo urbanos que, con diferentes énfasis en cada caso, mantienen bastantes rasgos comunes. Dentro de este mismo escenario interpretativo, el concepto de ciudad innovadora (innovative city), tal como aquí se plantea, toma prestadas algunas ideas de este tipo de propuestas pero aporta también especificidades y matices diferenciales en su interpretación que pueden quedar sintetizados en el esquema de la figura 1 .

De este modo, ciudades innovadoras serán aquellas donde se aplica una estrategia consciente y sistemática orientada a producir y transferir un recurso estratégico como es el conocimiento en sus diversas formas (teórico o práctico, científico o simbólico, analítico o sintético, explícito o tácito, etc.), acumulado en su población, sus empresas, sus instituciones públicas y sus organizaciones sociales. El objetivo será ampliar y activar ese stock de capital cognitivo y de capital humano para favorecer el desarrollo local. Para ello será necesario promover procesos de aprendizaje, tanto individual —a través del sistema educativo y de una mejora en el nivel formativo de la población - como colectivo - a través del trabajo conjunto e interactivo que favorece la transmisión de conocimiento tácito, difícil de codificar-, para lograr así un incremento de lo que algunos califican como inteligencia compartida.

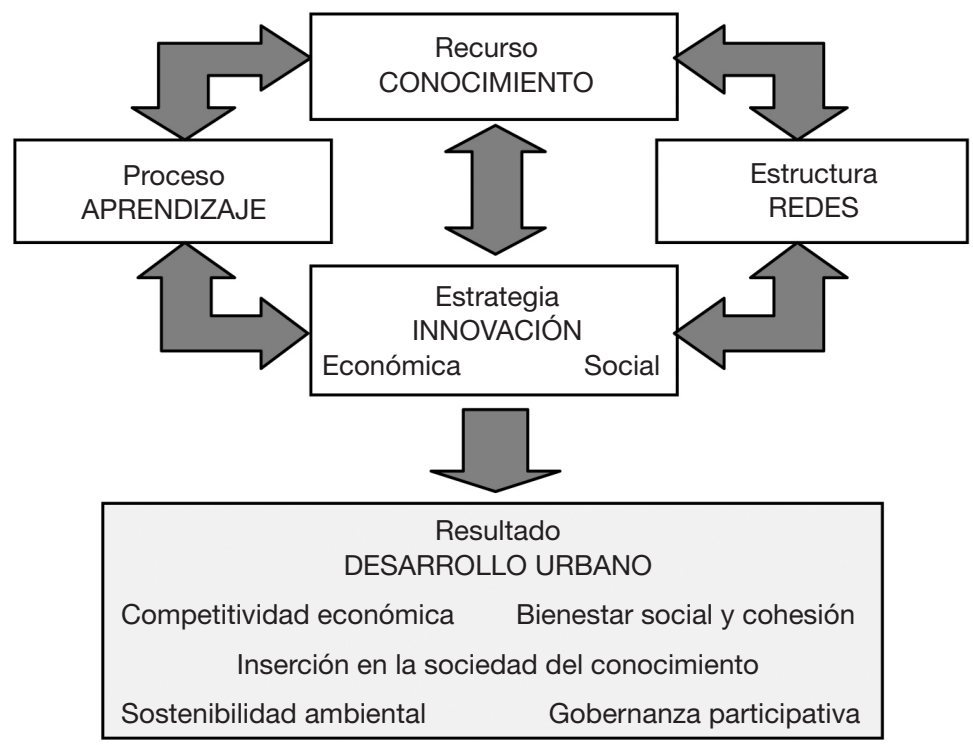

Figura 1. Ciudades innovadoras: componentes, estrategias y resultados.

Fuente: Elaboración propia. 
Para impulsar esos procesos, la organización más eficaz es la construcción de redes, formalizadas o de carácter informal, entre actores locales con características, estrategias e intereses diferentes, pero comprometidos en objetivos comunes (Caravaca y González, 2009). Al mismo tiempo, en un mundo globalizado que se caracteriza por la densificación de todo tipo de flujos, cobran creciente importancia las relaciones multiescalares que desde las ciudades se establecen con el exterior, tanto de sentido vertical como horizontal (Vale, 2011). El resultado de todo ello será la consolidación de procesos de innovación localizados, que hoy se consideran condición necesaria para el desarrollo urbano pero que, debido al uso abusivo del concepto, provocan cierta confusión sobre su verdadero significado desde la perspectiva de los estudios urbanos.

Conviene recordar que la teoría de la innovación surgió en el ámbito de la economía, y su traslación a los estudios territoriales - que destacaron sobre todo su carácter localizado - tuvo lugar dentro de la geografía económica, que la consideró un objetivo estratégico para elevar la competitividad de aquellos territorios capaces de incorporarla a una proporción creciente de su tejido empresarial. Desde esta perspectiva inicial, una empresa innovadora - y, por extensión, una economía urbana innovadora - será aquella que incremente la eficacia de sus procesos de trabajo, junto a la calidad y diferenciación de los bienes o servicios producidos, mediante la incorporación de mejoras tecnológicas, así como su organización y gestión internas, con lo que logrará también una apertura a mercados cada vez más amplios gracias a su creciente capacidad competitiva. Los estudios sobre modelos territoriales de innovación (Moulaert y Sekia, 2003), que aportaron conceptos como los de distrito industrial, ambiente innovador (milieu), sistema productivo local, clúster o sistema regional de innovación, pusieron en evidencia que las mayores tasas de innovación se alcanzaban en aquellos lugares donde empresas próximas que trabajan en torno al mismo tipo de productos mantienen estrechas relaciones de complementariedad y colaboración — también con gobiernos e instituciones ligadas al conocimiento- para lograr mayor eficiencia colectiva (Becattini et al., 2003; Braczyk et al., 2003; Camagni y Maillat eds., 2006...).

Pero existen ciudades que, pese a ser polos científico-tecnológicos y contar con numerosas empresas innovadoras, no necesariamente traducen eso en un desarrollo más inclusivo para sus ciudadanos, más sostenible en el plano ambiental, o en una gestión pública más eficaz. De ahí la necesidad de convertir también a las ciudades en ambientes socialmente innovadores, pues «la innovación es un proceso social para el que el lugar, la gente y las redes sociales resultan esenciales» (Maxwell, 2003: 9).

El concepto de innovación social aplicado al desarrollo urbano ha destacado la importancia de buscar nuevas respuestas para enfrentar los procesos de exclusión y fragmentación social, así como para promover la tolerancia ante la diversidad sociocultural y favorecer una participación más activa en procesos de debate y decisión ligados a la gestión urbana, que acumulen capital social y hagan posible la presencia en esos foros de los grupos sociales desfavorecidos 
(Fontan et al., 2004; André y Abreu, 2006; Klein y Harrison, 2007; MacCallum et al., 2009).

La acción combinada de las innovaciones económicas y sociales debería traducirse en una respuesta más eficaz ante los actuales retos del desarrollo, aspecto clave para valorar de forma positiva o no su verdadera aportación a la búsqueda de mejores soluciones para el presente y el futuro de las ciudades. Recordando ideas ampliamente difundidas, eso deberá reflejarse en un incremento de su competitividad económica y de la capacidad para generar más y mejores empleos, en una mayor cohesión social derivada de una amplia oferta de bienes públicos y una moderación de las desigualdades, así como en una gestión ambiental más sostenible. Todo lo anterior debería acompañarse también por formas de participación más abiertas que eviten una gobernanza sometida a la influencia hegemónica de determinadas élites locales. Un último componente del desarrollo urbano se asocia a una mejor inserción en la sociedad del conocimiento, lo que supone reforzar su dotación en capital humano, atraído por la presencia de ocupaciones cualificadas y una elevada calidad de vida, así como en instituciones educativas, culturales y relacionadas con la $\mathrm{I}+\mathrm{D}+\mathrm{i}$, junto a una buena conectividad exterior $\mathrm{y}$ de diferentes tipos de comunidades de aprendizaje.

\section{Ciudades medias e innovación: de las debilidades genéricas a las potencialidades específicas}

En un reciente libro dedicado a investigar las razones que cimentan el triunfo de las ciudades, Glaeser afirma que, «al igual que a las personas cualificadas, también parece que a las ciudades cualificadas se les da mejor reinventarse en tiempos volátiles» (Glaeser, 2011: 48). No obstante, la obra limita su atención a una serie de grandes ciudades-región que, al contar con una superior dotación de recursos materiales y humanos para promover el conocimiento, la creatividad y la innovación, suelen polarizar la atención de quienes se interesan por tales conceptos.

Sin negar esa tradicional primacía, en los últimos años ha comenzado a prestarse mayor atención a las ciudades medias, no solo por su función reequilibradora del territorio europeo, sino también porque se ha constatado la creciente capacidad de algunas para generar respuestas proactivas al reto de la globalización neoliberal y definir proyectos de futuro en los que la innovación está muy presente. Más allá de su tamaño poblacional, siempre en discusión y variable según contextos, buena parte de los análisis realizados en estos años han destacado su carácter de intermediarias potenciales entre las grandes aglomeraciones metropolitanas y los espacios rurales como proveedoras de bienes y servicios especializados para su entorno, lo que revalorizó su interés en relación con el objetivo de avanzar hacia un mayor policentrismo en la construcción europea (Bellet y Beltrão eds., 2009; Méndez dir., 2010). Pese a todo, buena parte de la investigación territorial sobre innovación apenas las considera, consciente de las debilidades que a este respecto aún mantienen buena parte de las ciudades de menor dimensión (figura 2). 
Entre esas debilidades genéricas en comparación con las grandes ciudades y regiones metropolitanas están, en primer lugar, las que suponen otros tantos obstáculos a la innovación en el plano económico. Aquí cabe mencionar la poca diversificación que a menudo caracteriza su economía y la dificultad para atraer a determinadas empresas por contar con infraestructuras de transporte y comunicación, así como con equipamientos, de segundo nivel. A eso se suma el neto predominio que suelen alcanzar las pymes y microempresas con escasa capacidad inversora y su frecuente especialización en actividades poco exigentes en capital humano o que realizan un limitado esfuerzo en innovación. Tal estructura sectorial converge con una escasa oferta de titulados superiores y trabajadores especializados, tanto por la habitual concentración de las universidades y otros centros de formación superior en las grandes urbes como por la poca disponibilidad de empleos de alta cualificación, lo que a menudo fuerza la marcha de una parte de los jóvenes mejor formados en busca de mayores oportunidades.

La escasez de instituciones vinculadas a la $\mathrm{I}+\mathrm{D}+\mathrm{i}$ acaba por limitar la capacidad del sistema local de innovación para generar y transferir mejoras a la economía de estas ciudades, dependientes de los procesos de difusión procedentes del exterior.

\section{INNOVACIÓN ECONÓMICA}

Debilidades tradicionales

- Limitada diversificación económica

- Infraestructuras físicas de segundo nivel

- Elevado \% de pymes/microempresas

- Escasez de clústeres innovadores

- Recursos humanos poco cualificados

- Déficit de instituciones de I + D + i
Nuevas oportunidades

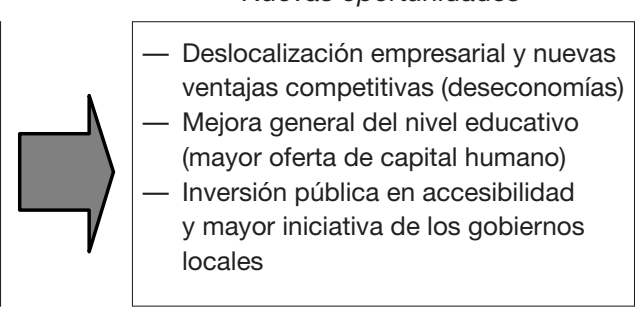

INNOVACIÓN SOCIAL

- Limitada diversidad social y cultural

- Escasez de iniciativas locales

- Déficit de equipamientos culturales

- Poca movilización ciudadana

- Gobiernos locales con escasos recursos e inercias burocráticas

- Escasa participación en redes de ciudades

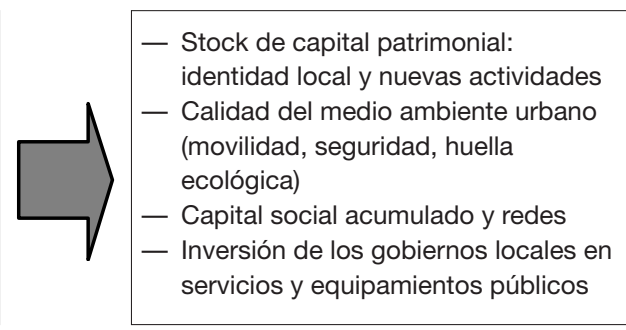

Figura 2. Debilidades tradicionales y nuevas oportunidades para la innovación en ciudades medias.

Fuente: Elaboración propia. 
Por su parte, en el plano de la innovación social son también frecuentes las alusiones a otros tantos frenos. La limitada diversidad sociocultural interna, unida a las inercias que algunos detectan en este tipo de sociedades locales, con un ambiente poco propicio al surgimiento de iniciativas, se traduciría en menores tasas de creatividad que en ámbitos metropolitanos más cosmopolitas, aspecto en el que los déficits de equipamientos culturales de calidad actúan como complemento. En este mismo plano de caracterización general, las frecuentes alusiones a la escasa movilización ciudadana y a la presencia de movimientos sociales que registran estas ciudades, junto con los limitados recursos de unos gobiernos locales más preocupados a menudo por la administración y la provisión de servicios básicos que por convertirse en gestores del desarrollo urbano mediante estrategias más arriesgadas e innovadoras, se añadirían a lo anterior para actuar como freno a su conversión en ciudades innovadoras.

Sin negar la permanencia de algunas de estas debilidades en numerosas ciudades medias, las nuevas lógicas socioeconómicas y territoriales emergentes en las últimas décadas han hecho surgir también nuevas oportunidades para superar algunas de estas carencias (figura 2). En primer lugar, en una economía cada vez más segmentada, algunas tareas antes concentradas en un mismo establecimiento dentro de las grandes ciudades-región encuentran ahora mejores condiciones para deslocalizarse hacia ciudades con menores deseconomías de aglomeración, pero bien conectadas y con mercados en expansión, lo que permite atraer hacia ellas establecimientos de empresas-red y actividades de mayor rango. La mejora general del nivel educativo y la descentralización universitaria han elevado en bastantes casos su stock de recursos humanos cualificados, y las importantes inversiones públicas en infraestructuras y equipamientos que han tenido a estas ciudades como destinatarias han supuesto una reducción de sus anteriores desventajas comparativas. La difusión de formas de gestión local que plantean el desarrollo económico como objetivo ha actuado también como factor de impulso a iniciativas dirigidas a insertar empresas locales en proyectos de innovación tecnológica y exploración de nuevos mercados, así como a promover nuevos espacios empresariales mejor equipados para localizar firmas más exigentes en materia de calidad del entorno.

Al mismo tiempo, las ciudades medias también se benefician hoy de una revalorización de su imagen por la elevada calidad de su medio ambiente urbano en términos de mayor seguridad y menor movilidad forzada o huella ecológica. También se presta ahora mayor atención a su stock de capital patrimonial, lo que favorece una mayor identificación de sus ciudadanos en relación con la que es habitual en las grandes metrópolis, lo que puede traducirse en una superior implicación respecto al futuro colectivo. Un tercer rasgo es la frecuente acumulación de capital social en bastantes de esas ciudades, base potencial para la construcción de redes de colaboración destinadas a plantear nuevas estrategias de desarrollo. Finalmente, la elevada inversión en servicios públicos ha favorecido cierto reequilibrio dotacional con respecto a las grandes urbes, al tiempo que en algunas se han explorado formas de gobernanza local 
más participativas, aunque de nuevo aquí se trata de tendencias generales con notorias diferencias según los casos.

\section{Ciudades medias frente a la crisis: innovación para la resiliencia urbana}

Pero si la innovación se considera importante para promover el desarrollo de todo tipo de ciudades medias, alcanza un significado estratégico en aquellas que, en el pasado reciente o en el presente, se enfrentan a situaciones de crisis estructural, causantes de un profundo y prolongado declive, a las que la bibliografía internacional identifica ahora como shrinking cities (Fol y CunninghamSabot, 2010).

Aunque desde el comienzo de la industrialización el crecimiento de las ciudades se convirtió en un fenómeno habitual, contrapuesto a la peor evolución de las áreas rurales, una revisión de la evolución urbana en Europa durante las últimas décadas constata que el declive está lejos de resultar algo anómalo. Ya en la primera mitad del pasado siglo, fueron bastantes las agrociudades y centros comarcales de servicios que interrumpieron su crecimiento anterior ante el declive de los territorios circundantes que les servían como soporte, sobre todo en la Europa mediterránea y las regiones interiores. En los años setenta y ochenta, en cambio, el agotamiento del modelo productivo fordista y el aumento de las deslocalizaciones empresariales asociadas a la nueva división internacional del trabajo provocaron el declive de numerosas ciudades industriales, portuarias y mineras, ahora localizadas sobre todo en la Europa atlántica. En la última década del siglo, la desaparición de la Unión Soviética, la profunda crisis de las economías de planificación centralizada y su precipitada conversión al capitalismo se saldaron con la contracción vivida por muchas ciudades de Europa central y oriental, en especial las que crecieron en torno a grandes combinats industriales ahora cerrados o privatizados y sometidos a intensos procesos de ajuste.

La crisis financiera internacional desatada en 2008, que provocó de forma inmediata la brusca detención del crecimiento económico en todas las ciudades europeas ante las restricciones al crédito, tanto privado como público, ha comenzado a producir impactos selectivos a partir de 2009, con el probable surgimiento de una nueva generación de shrinking cities, en bastantes ocasiones de tamaño medio (Soto, 2010), con una destacada presencia en la periferia europea. Aunque todavía está por hacer una investigación que profundice en el desigual comportamiento de las ciudades ante esta nueva crisis, su impacto parece ser mayor en las de aquellos países que, como España, conocieron en la década precedente un mayor crecimiento de la burbuja inmobiliaria y financiera (Aalbers, 2009; Naredo, 2009; Romero et al., 2012). En su interior, las ciudades que basaron lo esencial de su dinamismo reciente en el sector de la construcción, en las industrias y servicios auxiliares, o en una expansión incontrolada del consumo y los servicios a la población, con escasa productividad, empleo poco cualificado o precario y bajas tasas de innovación, parecen candidatas a situarse entre las más afectadas (Perlo, 2011). La recesión económica, 
el aumento del desempleo, la reaparición de saldos migratorios negativos o el hundimiento del mercado inmobiliario, junto al repunte de la pobreza y los fenómenos de exclusión social, agravados por los recortes en los presupuestos públicos impuestos por la agenda neoliberal dominante, resultan sus indicadores más visibles. A eso se unen la falta de expectativas, el pesimismo sobre el futuro y la frecuente inacción frente a la búsqueda de nuevas vías para la revitalización económica y la regeneración urbana, contexto en que surge ahora con fuerza la noción de resiliencia.

No se pretende abordar la diversidad de definiciones existentes para un concepto que, por esa razón, parece en ocasiones confuso. Tampoco la banalización que acompaña la incorporación de un término de moda, procedente de otro ámbito de estudio, cuando se le priva de su significado original, aspectos ya tratados en un texto anterior (Méndez, 2012). Desde la perspectiva aquí planteada, coherente con las propuestas de otros autores que la consideran a escala regional (Pike et al., 2010; Lang, 2011; Martin, 2012), la resiliencia urbana puede entenderse como la capacidad de adaptación positiva que muestran algunas ciudades para afrontar situaciones de crisis derivadas de acontecimientos o procesos externos que se ven reforzados por ciertas debilidades endógenas que las hicieron especialmente vulnerables y resurgir fortalecidas a partir de una estrategia de transformación interna. Si bien en algunos casos esas adversidades pueden corresponder a catástrofes acaecidas en momentos concretos, debidas a factores naturales o de origen humano (ciclones, sismos, tsunamis, bombardeos...), son más frecuentes las derivadas de crisis sistémicas que pusieron en cuestión las actividades y funciones en que se basó su crecimiento anterior, lo que plantea graves incertidumbres sobre su viabilidad futura.

La resiliencia corresponde, por tanto, a un proceso dinámico de trabajo consciente, destinado a lograr una mejor adaptación al nuevo contexto, con efectos sólo visibles a medio/largo plazo, que no resulta una cualidad inherente a todas las ciudades. Por el contrario, se hace presente tan solo en aquellas que, a partir de un diagnóstico realista sobre sus limitaciones y potencialidades, son capaces de movilizar recursos y emprender acciones para superar inercias y bloqueos heredados que lastran su recuperación y aplicar estrategias de innovación económica y social que permitan encontrar nuevas respuestas ante la crisis. No se trata, por tanto, de pretender el regreso a modelos de crecimiento que se muestran ya agotados, pero tampoco de proponer objetivos quiméricos que ignoren sus potencialidades y limitaciones.

Si la resiliencia supone reiniciar la senda del desarrollo, los indicadores que pueden servir para identificar a aquellas ciudades que han iniciado ese camino serán los mismos utilizados de forma habitual para valorar el desarrollo urbano. Recuperar, por tanto, el crecimiento de la actividad económica y la generación de empleo, un balance migratorio positivo, una mejora de los niveles de bienestar social y de los servicios públicos destinados a ese fin, o una renovación del tejido urbano pueden ser algunos de los más evidentes.

La resiliencia urbana así entendida no puede basarse solo en la ayuda externa, sino que exige combinar políticas de apoyo generadas en instancias supe- 
riores con iniciativas locales, en una perspectiva multiescalar (CSIRO, 2007). Tampoco puede ignorar el pasado, sino que existe una dependencia de la trayectoria (Simmie y Martin, 2010) que obliga a aprender de lo ocurrido, poner en valor aquellos recursos específicos de que se dispone y que, renovados, sigan siendo viables, e invertir en aquellos otros considerados estratégicos para superar carencias locales, lo que supone ser crítico con la simple importación de modelos de éxito en otras ciudades.

No existe, pues, una única vía para avanzar en el objetivo de ser una ciudad resiliente, ni se trata de un logro que, una vez alcanzado, resulte duradero. Por el contrario, cada ciudad deberá explorar entre alternativas posibles y mantener un esfuerzo de adaptación a entornos tan cambiantes como los actuales. También buscar la concertación en torno a un proyecto colectivo que resulte movilizador, aspecto especialmente difícil allí donde el desigual reparto de los costes de la crisis aumenta la desconfianza y los conflictos internos.

Todo lo expuesto hasta el momento suscita una pregunta esencial, que solo puede encontrar respuesta en estudios sobre ciudades concretas: ¿por qué algunas ciudades medias muestran más capacidad que otras para avanzar por el camino de la innovación y, en su caso, enfrentarse de forma proactiva a los efectos de una crisis? A partir de casos analizados pueden apuntarse algunas respuestas a modo de hipótesis, que deberían ser contrastadas en un más amplio programa de investigación colectivo que permita profundizar y matizar unas afirmaciones aún demasiado generales.

\section{Claves para la construcción de ciudades medias innovadoras y resilientes}

La observación de ciudades medias que han sido capaces de incrementar su esfuerzo innovador para superar el declive muestra claras diferencias en cuanto a la metodología utilizada o la trayectoria seguida, lo que excluye la existencia de un modelo único a este respecto. También debe tenerse en cuenta que los estudios disponibles analizan procesos ocurridos en el pasado reciente en ciudades que se enfrentaron a crisis con orígenes y rasgos distintos a la actual. Pese a todo, permiten deducir algunas enseñanzas que van más allá de cada caso específico y ofrecen una buena base de partida para la reflexión. En el caso español existen algunos que abordan estas cuestiones desde una perspectiva teórica próxima a la aquí propuesta - aunque sin referencia directa al concepto de resiliencia-, tanto en ciudades ubicadas en entornos metropolitanos (Méndez, García Palomares y Michelini, 2007; Caravaca y González, 2010), como en núcleos de antigua tradición industrial y minera (Tomé, 2010; Sánchez Moral, Méndez y Prada, 2012) o que perdieron su anterior funcionalidad como nodos ferroviarios (Michelini, 2010).

Puede proponerse un esquema interpretativo útil para abordar esas posibles claves a partir de ideas como las propuestas por Pelling y Manuel-Navarrete (2011), considerando que la capacidad de adaptación urbana comienza por ser capaz de identificar nuevos objetivos en un proyecto de futuro que permita reinventar la ciudad, a lo que deberá sumarse una nueva forma de gobernanza 
capaz de gestionarlo, así como nuevos instrumentos y formas de actuación que materialicen lo anterior.

El primer punto de encuentro entre las ciudades que se han enfrentado con relativo éxito a una crisis parece ser la presencia de individuos y organizaciones, tanto en el sector público como en el ámbito de las empresas locales o la sociedad civil, con voluntad y capacidad para generar iniciativas novedosas - al menos en la evolución de esa ciudad - destinadas a recuperar su competitividad, al tiempo que la calidad de vida de sus ciudadanos, sin limitarse a plantear respuestas defensivas o esperar soluciones procedentes del exterior. Esos actores urbanos son protagonistas necesarios de cualquier estrategia de dinamización que pueda emprenderse, pero también pueden suponer su principal obstáculo cuando su falta de iniciativa, de recursos (financieros, de conocimiento...), o la defensa de intereses privados de determinadas élites en contradicción con los colectivos acaban por imponerse.

En ámbitos de cierta complejidad como son las ciudades medias, es importante que los proyectos dinamizadores, a menudo asociados en su origen a personas concretas, evolucionen del individuo a la organización para asegurar que perduran en el tiempo y se generen procesos acumulativos a medio plazo, cuestión no resuelta cuando tales iniciativas siguen estando muy personalizadas. También se constata la importancia de que exista un liderazgo ejercido por el gobierno local, basado en cierto grado de credibilidad, confianza y autoridad, al menos desde dos perspectivas complementarias. Por una parte, en la definición de objetivos colectivos orientados a promover la innovación, compatibles con los de la mayoría de grupos sociales y corporativos presentes en la ciudad pero no sometidos a ninguno de ellos. Por otra, como agente catalizador capaz de dialogar y tejer vínculos de confianza entre los restantes actores -internos y externos a la ciudad - para promover foros de concertación, debate y negociación del conflicto, ya sea en el marco de un plan de ordenación urbana, un plan estratégico o un pacto local, función que sigue siendo esencial en tiempos en que los recursos financieros de las corporaciones locales escasean.

Esto último plantea la importancia del marco institucional local en su doble dimensión cultural y organizativa. La presencia de valores, convenciones y reglas de comportamiento favorables al surgimiento de innovaciones o al trabajo colectivo, muy diversa según la trayectoria histórica de cada ciudad, actúa como recurso potencial que puede movilizarse en algunos casos a favor de los objetivos señalados. Pero, más allá de ese ambiente heredado, el capital social puede generarse y acumularse mediante la promoción de redes de cooperación entre actores locales que a su proximidad física añadan la cultural, derivada de códigos y lenguajes comunes. Hace dos décadas, la atención se centraba en las redes locales de empresas que mantenían entre sí densas relaciones de carácter mercantil (vínculos proveedor-cliente, subcontratación...), o al margen del mercado (intercambio de información, proyectos conjuntos...), lo que generaba un cierto volumen de externalidades. Más tarde, el interés se amplió a todo tipo de redes sociales capaces de construir comunidades de aprendizaje localizadas, como medio para superar un individualismo excesivo que dificulta 
a menudo la transferencia de conocimiento tácito e impide sumar capacidades (Salom y Albertos eds., 2009).

Todo esto se vincula también con el concepto de gobernanza urbana en cuanto forma de gestión concertada de las ciudades que permite construir coaliciones de actores, tanto públicos como privados, para promover su desarrollo. Si bien una gobernanza local más participativa puede generar efectos positivos al aumentar el volumen de recursos aportados para la innovación y legitimar en mayor medida las decisiones adoptadas (Pascual y Godás, 2010), la evidencia de que pueden construirse diferentes tipos de regímenes urbanos (Casellas, 2005) que identifican otras tantas coaliciones hegemónicas pone en guardia contra visiones a veces demasiado simplificadas respecto a sus efectos. Tal como ha señalado Swyngedouw (2005), en determinadas ciudades unas relaciones de poder desequilibradas entre intereses contrapuestos pueden saldarse con la formación de coaliciones locales donde tan solo estén representadas determinadas élites, lo que además de suponer un déficit democrático puede provocar un sesgo en cuanto al tipo de innovación que se promueve e ignorar la atención a los grupos sociales y espacios más golpeados por la crisis.

Esto último nos recuerda que el tercer componente habitual en ciudades medias que han demostrado capacidad de innovación y de enfrentarse a las crisis es la puesta en práctica de estrategias y políticas planteadas con esos objetivos. Se trata con ellas de incrementar los recursos específicos locales, en especial el capital humano, social e intelectual, aunque sin olvidar la inversión en capital físico bajo la forma de infraestructuras y equipamientos que faciliten la innovación, pero desplazando la importancia prioritaria tradicionalmente concedida a las políticas duras (hard policies) en beneficio de las políticas blandas (soft policies). Sin olvidar la necesidad de mantener activo todo un conjunto de estrategias inclusivas en lo social y establecer criterios de ordenación urbanística frente a la desregulación, las que aquí interesa destacar son las dirigidas a potenciar la innovación económica y social, además del esfuerzo de inserción exterior, esencial en tiempos como los actuales (figura 3).

En el primero de esos planos el apoyo debe comenzar en la fase previa al nacimiento de las empresas y mantenerse activo, sobre todo, durante las primeras fases de su ciclo de vida mediante el asesoramiento o la oferta de viveros y centros de empresas para albergarlas de forma temporal. Incluye también diversas acciones para mejorar la eficiencia y competitividad que sean asumibles desde la escala local: creación de una oferta formativa adaptada a las específicas necesidades y posibilidades existentes en la ciudad, ayudas a la formación de clústeres locales que desarrollen proyectos en común (desde centrales de compras hasta campañas de promoción exterior), instalación de centros tecnológicos, mejora de las infraestructuras digitales, etc. Se trata de actuaciones para favorecer la innovación, pero que ya no resultan demasiado innovadoras en sí mismas debido a su amplia difusión, a diferencia de los centros o fábricas de creación, dirigidos a albergar a emprendedores vinculados a segmentos de las industrias culturales relacionados con las nuevas tecnologías (diseño digital, 


\section{INNOVACIÓN ECONÓMICA}

- Asesoramiento a emprendedores, pymes y microempresas.

- Apoyo a la creación de clústeres locales de empresas.

- Formación especializada (adecuación a demanda local).

- Promoción de espacios para fomentar la innovación (viveros, centros de empresas, centros tecnológicos, fábricas de creación).

— Infraestructuras digitales de alta capacidad...

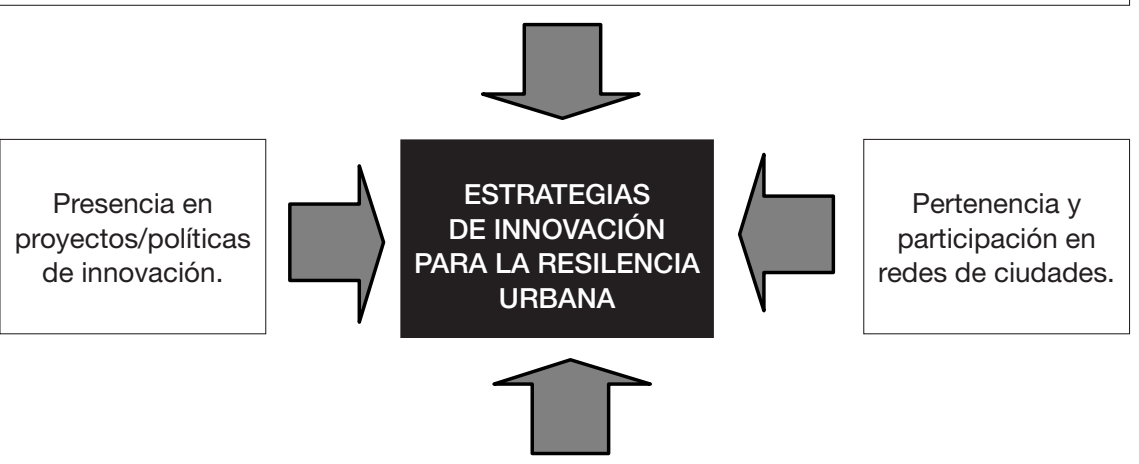

INNOVACIÓN SOCIAL

- Creación de foros estables de participación.

- Fomento de la identidad urbana (calidad, diversidad, tolerancia...).

- Aumento de dotación en bienes y equipamientos culturales.

- Protección y valorización del patrimonio.

- Renovación de áreas deterioradas y regeneración ambiental.

Figura 3. Estrategias de promoción de la innovación en ciudades medias.

Fuente: Elaboración propia.

videojuegos, multimedia...), que ocupan en ocasiones inmuebles abandonados y se integran en estrategias de renovación de barrios (Winden, 2011).

Desde la perspectiva de la innovación social puede mencionarse la creación de foros estables donde los diferentes actores urbanos, incluidos aquellos movimientos ciudadanos que representan a grupos desfavorecidos o especialmente afectados por la crisis, puedan participar, junto a todas aquellas actuaciones que incidan en ligar la identidad y la imagen urbana a valores como la calidad, la diversidad o la tolerancia. Las mejoras dotacionales en bienes culturales y en la defensa y puesta en valor del patrimonio resultan coherentes con este tipo de acciones, al igual que las estrategias de regeneración de áreas deterioradas para dotarlas de una nueva capacidad de acogida.

\section{Conclusiones}

Aunque cada ciudad es heredera de su propio pasado y debe enfrentarse a la búsqueda de caminos para su desarrollo en entornos espaciotemporales también específicos, los estudios sobre innovación territorial y sobre resiliencia 
urbana pueden suponer hoy una llamada de atención sobre las claves para entender por qué y cómo algunas ciudades lograron superar serias dificultades en su pasado reciente y avanzar hacia un mayor y mejor desarrollo, no solo en lo económico sino también desde una perspectiva más integrada y compleja.

En las páginas anteriores se ha destacado la idea de que, desde la perspectiva del desarrollo urbano, la innovación no puede limitarse a considerar la capacidad científico-técnica o el potencial de las empresas locales para hacer más eficientes sus procesos y su organización y mejores sus productos o sus formas de acceso a nuevos mercados. Junto a estas dimensiones de la innovación, es necesario considerar también el fomento de la innovación social, entendida como la capacidad para lograr mejoras tangibles en la calidad de vida, la participación ciudadana, la protección del patrimonio o la sostenibilidad social y ambiental.

Las ciudades medias, muy numerosas en la mayoría de sistemas urbanos, son lugares estratégicos para la recuperación económica y el reequilibrio territorial. Resultan tradicionales sus desventajas comparativas respecto a las grandes áreas urbanas en materia de dotación de recursos materiales y humanos, en especial los que hacen un uso más intensivo del conocimiento. Pero, si se considera la innovación desde el enfoque aquí propuesto, se constata que en las últimas décadas han surgido nuevas oportunidades para las ciudades medias y, sobre todo, que algunas de ellas han demostrado una elevada capacidad para renovarse y afrontar situaciones de crisis, hasta convertirse en exponentes de la denominada resiliencia urbana.

Aunque no existe una única vía para promover un mayor esfuerzo innovador que conduzca a un mejor desarrollo y, en algunos casos, a la recuperación de ciudades en declive por el agotamiento de su modelo de crecimiento anterior, sí parecen existir ciertos procesos comunes y las ciudades medias resultan un laboratorio especialmente adecuado para comprender mejor sus claves explicativas y las políticas más adecuadas en esa dirección. Construir una gobernanza relacional entre actores locales que mantenga el liderazgo del gobierno de la ciudad y evite la usurpación de la representación ciudadana por determinadas élites supone una base de partida. Plantear alternativas que tomen en consideración la trayectoria urbana para evitar saltos en el vacío o la simple imitación de estrategias aplicadas en otras ciudades resulta un segundo aspecto que constata el significado de la llamada path dependence. Combinar estrategias que tengan en cuenta el fomento tanto de la innovación empresarial como de la innovación social resulta un tercer criterio para diferenciar lo ocurrido en ciudades de tamaño y entorno regional similar, pero que muestran notorias diferencias en su evolución reciente.

En momentos como el actual, que enfrenta a numerosas ciudades medias de Europa con serias incertidumbres sobre su futuro y refuerza la necesidad de explorar caminos que permitan superar el pesimismo y la inacción, no está de más recordar que "en toda sociedad existen posibilidades específicas para una mejora de la vida humana y formas y medios específicos para realizar esas posibilidades» (Marcuse, 1985: 21). La investigación social y territorial puede 
resultar útil para conocer y comprender mejor cómo y por qué algunos lugares han sido capaces de convertir ese potencial en formas de desarrollo urbano más equilibradas, justas y sostenibles, cuestión que seguirá siendo relevante desde el punto de vista científico y social en los próximos años.

\section{Referencias bibliográficas}

AAlBeRS, M. (2009). «The Sociology and Geography of Mortgage Markets: Reflections on the Financial Crisis». International Journal of Urban and Regional Research, 33 (2), 281-90.

ANDRÉ, I. Y ABReu, A. (2006). «Dimensōes e espaços da inovação social». Finisterra, XLI (81), 121-41.

Becattini, G.; Bellandi, M.; Dei Otatti, G. y Sforzi, F. (2003). From Industrial Districts to Local Development. An Itinerary of Research. Cheltenham: Edward Elgar.

BELLET, C. y BELTRĀo, E. (eds.) (2009). Las ciudades medias o intermedias en un mundo globalizado. Lérida: Edicions Universitat de Lleida.

BraczyK, H. J.; CoOKe, P. y HeIdEnReICH, M. (eds.) (2003). Regional Innovation Systems. Londres: Routledge.

Camagni, R. y Maillat, D. (eds.) (2006). Milieux innovateurs. Théorie et politiques. París: Economica-Anthropos.

Caragliu, A; Del Bo, C. y Nijkamp, P. (2009). «Smart cities in Europe». Serie Research Memoranda 0048, Amsterdam: VU University Amsterdam.

CARAVACA, I. y GonZÁlez, G. (2009). "Las redes de colaboración como base del desarrollo territorial». Scripta Nova, XIII, 289.

- (2010). «Estrategias y actuaciones para el desarrollo de ciudades medias. Algunos ejemplos». Scripta Nova, XIV, 331.

CASElLAS, A. (2005). «Aproximaciones teóricas para el análisis de la transformación urbana: Urban Regime Analysis en el contexto europeo». Documents d'Anàlisi Geogràfica, 46, 139-47.

COMISIÓN EUROPEA (2010). Europa 2020. Una estrategia para un crecimiento inteligente, sostenible e integrador. Bruselas: Comisión Europea.

CSIRO (2007). A research prospectus for urban resilience. A Resilience Alliance Initiative for transitioning urban systems towards sustainable futures. Canberra: CSIRO - Arizona State University - Stockolm University.

FloRIDA, R. (2005): Cities and the creative class. Nueva York: Routledge.

Fol, S. y CUNNINGHAM-SABOT, E. (2010). «Declin urbain et shrinking cities: une evaluation critique des approches de la décroissance urbaine». Annales de Géographie, 674, 359-83.

Fontan, J. M.; Klein, J. L. y Tremblay, D. G. (2004). «Innovation et société : pour élargir l'analyse des effets territoriaux de l'innovation». Géographie, Économie, Société, 6, 115-28.

GLAESER, E. (2011). Triumph of the city. How our greatest invention makes us richer, smarter, greener, healthier and happier. Nueva York: The Penguin Press (Traducción española: El triunfo de las ciudades. Madrid: Taurus, 2011).

Hall, P. (2000). «Creative cities and economic development». Urban Studies, 37 (4), 639-49.

KleIN, J. L. y HarRIsOn, D. (eds.) (2007). L'innovation sociale. Émergence et effets sur la transformation des societés. Quebec: Presses de l'Université du Québec. 
Komninos, N. (2002). Intelligent Cities: Innovation, Knowledge Systems and Digital Spaces. Londres: Sponpress.

LANG, T. (2011). "Urban resilience and new institutional theory. A happy couple for urban and regional studies?». En: B. Müller (ed.). Urban regional resilience: how do cities and regions deal with change? Berlín-Heidelberg: Springer Verlag, 15-24.

Maccallum, D.; Moulaert, F. y Hillier, F. (2009). Social innovation and territorial development. Londres: Ashgate.

Marcuse, H. (1954). One-dimensional man. Boston: Bacon Press (Traducción española: El hombre unidimensional. Barcelona: Planeta, 1985).

MARTIN, R. (2012). «Regional economic resilience, hysteresis and recessionary shocks». Journal of Economic Geography, 12 (1), 1-32.

MAXWELl, J. (2003). "Innovation is a social process». Canada's National Statistical Agency. <http://www.statcan.ca/english/research/88F0006XIE/88F0006X IE2003006.pdf $>$.

MÉNDEZ, R. (dir.) (2010). Estrategias de innovación industrial y desarrollo económico en las ciudades intermedias de España. Madrid: Fundación BBVA.

- (2012). "Ciudades y metáforas: sobre el concepto de resiliencia urbana». Ciudady Territorio Estudios Territoriales, XLIV, 172.

Méndez, R.; García Palomares, J. C. y Michelini, J. J. (2007). «La construcción de ciudades innovadoras: transformaciones económicas recientes y desarrollo local en Getafe». Boletín de la Asociación de Geógrafos Españoles, 42, 7-30.

Michelini, J. J. (2010). «Gobernanza territorial, iniciativas locales y desarrollo urbano: los casos de Getafe y Alcázar de San Juan». Boletín de la Asociación de Geógrafos Españoles, 54, 175-201.

MoulaerT, F. y SeKIA, F. (2003). "Territorial innovation models: a critical survey». Regional Studies, 37 (3), 289-302.

NAREDO, J. M. (2009). «La cara oculta de la crisis. El fin del boom inmobiliario y sus consecuencias». Revista de Economía Crítica, 7, 313-40.

OCDE (2001). Cities and regions in the new learning economy. París: OCDE.

PASCUAL, J. M. y GODÁs, X. (coords.) (2010). El buen gobierno 2.0: la gobernanza democrática territorial. Valencia: Tirant lo Blanch.

Pelling, M. y Manuel-Navarrete, D. (2011). «From resilience to transformation: the adaptative cycle in two Mexican urban centres». Ecology and Society, 16 (2), 11. <http://www.ecologyandsociety.org/vol16/iss2/art11>

Perlo, M. (2011). "Cities in times of crisis. The response of local governments in light to the global economic crisis: the role of the formation of human capital, urban innovation and strategic planning». IURD Working Paper 2011-01. Berkeley Institute of Urban and Regional Development.

PIKe, A.; DaWley, S. y TOMAneY, J. (2010). "Resilience, adaptation and adaptability». Cambridge Journal of Regions, Economy and Society, 3, 59-70.

ROMERO, J.; JimÉNEZ, F. y VilloriA, M. (2012). «(Un)sustainable territories: causes of the speculative bubble in Spain (1996-2010) and its territorial, environmental and sociopolitical consequences». Environment and Planning C, 30, 467-86.

Salom, J. y Albertos, J. M. (eds.) (2009). Redes socioinstitucionales, estrategias de innovación y desarrollo territorial en España. Valencia: Publicaciones de la Universidad de Valencia.

Sánchez Moral, S.; Méndez, R. y Prada, J. (2012). "Avilés, entre el declive y la revitalización: ¡en la génesis de un nuevo modelo de desarrollo? Boletín de la Asociación de Geógrafos Españoles, 60, 321-48. 
Simmie, J. y Martin, R. (2010). «The economic resilience of regions: towards an evolutionary approach». Cambridge Journal of Regions, Economy and Society, 3, 27-43.

SotO, P. (2010). Cities at the economic crisis. A survey on the impact of the economic crisis and the responses of URBACT II cities. Bruselas: Comision Europea.

SWYNGEDOUW, E. (2005). "Governance innovation and the citizen: the Janus face of governance-beyond-the-State». Urban Studies, 42 (11), 1991-2006.

Tomé, S. (2010). «Langreo, Mieres, Ponferrada, Puertollano: cambios funcionales y morfológicos en ciudades minero-industriales». Scripta Nova, XIV, 336 (14).

VALE, M. (2011). "Innovation networks and local and regional development policy». En: Pike, A.; Rodríguez-Pose, A. y Tomaney J. (eds.). Handbook of local and regional development. Nueva York: Routledge, 413-24.

WINDEN, W. VAN (2011). Creating knowledge hotspots in the city: a handbook. Bruselas: Comisión Europea, URBACT II-REDIS.

Winden, W. van; BerG, L. VAN DEN y POL, P. (2007). "European cities in the knowledge economy: towards a tipology», Urban Studies, 44 (3), 525-49. 